\title{
Generalized cyclic contractions and coincidence points involving a control function on partial metric spaces
}

\author{
Sushanta Kumar Mohanta
}

Department of Mathematics, West Bengal State University, Kolkata, India

\begin{abstract}
Purpose - In this paper, we use the notion of cyclic representation of a nonempty set with respect to a pair of mappings to obtain coincidence points and common fixed points for a pair of self-mappings satisfying some generalized contraction- type conditions involving a control function in partial metric spaces. Moreover, we provide some examples to analyze and illustrate our main results.

Design/methodology/approach - Theoretical method.

Findings - We establish some coincidence points and common fixed point results in partial metric spaces.

Originality/value - Results of this study are new and interesting.
\end{abstract}

Keywords Partial metric, Cyclic contraction, 0-Completeness, Coincidence point

Paper type Research paper

\section{Introduction}

In 1994, Matthews [1] introduced the concept of partial metric spaces as a part of the study of denotational semantics of dataflow networks and proved the well-known Banach contraction principle in this setting. Complete partial metric space is a useful framework to model several complex problems in theory of computation. The works of [2-10] are viable and have opened new avenues for application in different fields of mathematics and applied sciences. It is interesting to note that in partial metric spaces self-distance of an arbitrary point need not be equal to zero. Recently, many authors studied fixed points of cyclic mappings in several spaces. In 2003, Kirk et al. [11] introduced the notion of cyclic mappings and proved some fixed-point theorems for these mappings. Some results for cyclic contractions in partial metric spaces have been obtained in [12-16]. In many cases, new results are being obtained by considering contractive conditions that depend on control functions. Indeed, the auxiliary functions which involved in contractive-type conditions are known as control functions. In 2013, Shatanawi et al. [17] proved some common fixed-point theorems with the help of control functions, namely, altering distance functions due to Khan et al. [18]. After that, several generalized control functions were used to obtain fixed-point results in various spaces. The results of [19-22] have become the source of motivation of this study. In this work, we introduce the concept of cyclic representation of a nonempty set with respect to a pair of mappings and use it to prove a coincidence point and common fixed-point result for a pair of self-mappings satisfying some generalized contraction-type conditions involving a control

\section{MSC Classification - 54H25, 47H10}

(C) Sushanta Kumar Mohanta. Published in Arab Journal of Mathematical Sciences. Published by Emerald Publishing Limited. This article is published under the Creative Commons Attribution (CC BY 4.0) licence. Anyone may reproduce, distribute, translate and create derivative works of this article (for both commercial and non-commercial purposes), subject to full attribution to the original publication and authors. The full terms of this licence may be seen at http://creativecommons.org/licences/by/4.0/ legalcode

The author is grateful to the referees for their valuable comments.

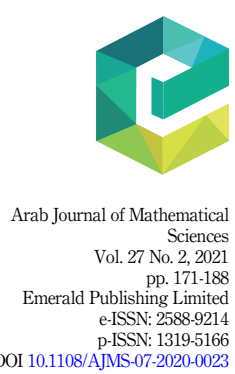


AJMS

27,2

function in partial metric spaces. We also prove another common fixed point result for a pair of self-mappings satisfying a new contraction condition in this framework. Our results extend and unify several existing results in the literature. Finally, we give some examples to justify the validity of our results.

\section{Some basic concepts}

In this section, we present some basic facts and properties of partial metric spaces.

Definition 2.1. [1] A partial metric on a nonempty set $X$ is a function $p: X \times X \rightarrow \mathbb{R}^{+}$such that for all $x, y, z \in X$ :

$$
\begin{gathered}
\left(p_{1}\right) p(x, x)=p(y, y)=p(x, y) \Leftrightarrow x=y, \\
\left(p_{2}\right) p(x, x) \leq p(x, y), \\
\left(p_{3}\right) p(x, y)=p(y, x), \\
\left(p_{4}\right) p(x, y) \leq p(x, z)+p(z, y)-p(z, z) .
\end{gathered}
$$

The pair $(X, p)$ is called a partial metric space.

It is obvious that if $p(x, y)=0$, then from $\left(p_{1}\right)$ and $\left(p_{2}\right)$, it follows that $x=y$. However, $x=y$ does not imply $p(x, y)=0$.

Example 2.2. [1]Let $X=[0, \infty)$ and let $p(x, y)=\max \{x, y\}$, for all $x, y \in X$. Then $(X, p)$ is a partial metric space.

Example 2.3. [1] Let $X=\{[a, b]: a, b \in \mathbb{R}, a \leq b\}$ and let $p([a, b],[c, d])=(\max$ $\{b, d\}-\min \{a, c\})$. Then $(X, p)$ is a partial metric space.

Each partial metric $p$ on $X$ generates a $T_{0}$ topology $\tau_{p}$ on $X$ which has as a base the family of open $p$-balls $\left\{B_{p}(x, \epsilon): x \in X, \epsilon>0\right\}$, where $B_{p}(x, \epsilon)=\{y \in X: p(x, y)<p(x, x)+\epsilon\}$ for all $x \in X$ and $\epsilon>0$.

Theorem 2.4. If $U \in \tau_{p}$ and $x \in U$, then there exists $r>0$ such that $B_{p}(x, r) \subseteq U$.

Proof. Since $U$ is an open set containing $x$, there exists an open $p$-ball, say $B_{p}(y, \epsilon)$ such that $x \in B_{p}(y, \epsilon) \subseteq U$. Then $p(x, y)<p(y, y)+\epsilon$. Let us choose $0<r<p(y, y)-p(x, y)+\epsilon$ and consider the open $p$-ball $B_{p}(x, r)$. Then it is easy to verify that $B_{p}(x, r) \subseteq B_{p}(y, \epsilon) \subseteq U$.

Remark 2.5. Let $(X, p)$ be a partial metric space, $\left(x_{n}\right)$ be a sequence in $X$ and $x \in X$. Then $\left(x_{n}\right)$ converges to $\mathrm{x}$ with respect to (w.r.t.) $\tau_{p}$ if and only if $\lim _{n \rightarrow \infty} p\left(x_{n}, x\right)=p(x, x)$.

Let $x_{n} \rightarrow x$ w.r.t. $\tau_{p}$ and $\epsilon>0$. Then there exists a natural number $n_{0}$ such that $x_{n} \in B_{p}(x, \epsilon)$ for all $n \geq n_{0}$. This gives that $p\left(x_{n}, x\right)-p(x, x)<\epsilon$ for all $n \geq n_{0}$. Since $p\left(x_{n}, x\right)-p(x, x) \geq 0$, it follows that $\left|p\left(x_{n}, x\right)-p(x, x)\right|<\epsilon$ for all $n \geq n_{0}$. This proves that $\lim _{n \rightarrow \infty} p\left(x_{n}, x\right)=p(x, x)$.

Conversely, suppose that $\lim _{n \rightarrow \infty} p\left(x_{n}, x\right)=p(x, x)$. We shall show that $x_{n} \rightarrow x$ w.r.t. $\tau_{p}$. Let $U \in \tau_{p}$ and $x \in U$. Then there exists $\epsilon>0$ such that $x \in B_{p}(x, \epsilon) \subseteq U$. By hypotheses, it follows that

$$
\lim _{n \rightarrow \infty}\left(p\left(x_{n}, x\right)-p(x, x)\right)=0 .
$$

So, there exists $n_{0} \in \mathbb{N}$ such that $p\left(x_{n}, x\right)-p(x, x)<\epsilon$ for all $n \geq n_{0}$. This ensures that $x_{n} \in B_{p}(x, \epsilon)$ for all $n \geq n_{0}$, and hence $x_{n} \in U$ for all $n \geq n_{0}$. Therefore, $\left(x_{n}\right)$ converges to $x$ w.r.t. $\tau_{p}$ on $X$. 
Definition 2.6. $[1]$ Let $(X, p)$ be a partial metric space, and let $\left(x_{n}\right)$ be a sequence in $X$. Then

(1) $\left(x_{n}\right)$ converges to a point $x \in X$ if $\lim _{n \rightarrow \infty} p\left(x_{n}, x\right)=p(x, x)$. This will be denoted as $\lim _{n \rightarrow \infty} x_{n}=x$ or $x_{n} \rightarrow x(n \rightarrow \infty)$.

(2) $\left(x_{n}\right)$ is called a Cauchy sequence if $\lim _{n, m \rightarrow \infty} p\left(x_{n}, x_{m}\right)$ exists and is finite, say $l$, that is, corresponding to every $\epsilon>0$, there exists $n_{0} \in \mathbb{N}$ such that $\left|p\left(x_{n}, x_{m}\right)-l\right|<\epsilon$, $\forall n, m \geq n_{0}$.

(3) $(X, p)$ is said to be complete if every Cauchy sequence $\left(x_{n}\right)$ in $X$ converges to a point $x \in X$ such that $p(x, x)=\lim _{n, m \rightarrow \infty} p\left(x_{n}, x_{m}\right)$.

Definition 2.7. [23] A sequence $\left(x_{n}\right)$ in $(X, p)$ is called 0-Cauchy if

$$
\lim _{n, m \rightarrow \infty} p\left(x_{n}, x_{m}\right)=0
$$

The space $(X, p)$ is said to be 0 -complete if every 0-Cauchy sequence in $X$ converges to a point $x \in X$ such that $p(x, x)=0$.

It is easy to verify that every closed subset of a 0-complete partial metric space is 0-complete.

Lemma 2.8. Let $(X, p)$ be a partial metric space.

(1) (see [24, 25]) If $p\left(x_{n}, z\right) \rightarrow p(z, z)=0$ as $n \rightarrow \infty$, then $p\left(x_{n}, y\right) \rightarrow p(z, y)$ as $n \rightarrow \infty$ for each $y \in X$.

(2) (see [23]) If $(X, p)$ is complete, then it is 0-complete.

The converse assertion of $(b)$ may not hold, in general. The following example supports the above remark.

Example 2.9. [23] The space $X=[0, \infty) \cap \mathbb{Q}$ with the partial metric $p(x, y)=\max \{x, y\}$ is 0-complete, but it is not complete. Moreover, the sequence $\left(x_{n}\right)$ with $x_{n}=1$ for each $n \in \mathbb{N}$ is a Cauchy sequence in $(X, p)$, but it is not a 0 -Cauchy sequence.

Definition 2.10. [26] Let $f$ and $g$ be self-mappings of a set $X$. If $y=f x=g x$ for some $x$ in $X$, then $x$ is called a coincidence point of $f$ and $g$ and $y$ is called a point of coincidence of $f$ and $g$.

Definition 2.11. [25] The mappings $f, g: X \rightarrow X$ are weakly compatible, if for every $x \in X$, the following holds:

$$
f(g x)=g(f x) \text { whenever } g x=f x .
$$

Proposition 2.12. [26] Let $f$ and $g$ be weakly compatible self-maps of a nonempty set $X$. If $f$ and $g$ have a unique point of coincidence $y=f x=g x$, then $y$ is the unique common fixed point of $f$ and $g$.

Let $\Psi$ be a class of functions $\psi:[0, \infty) \rightarrow[0, \infty)$ satisfying the following conditions:

$\left(\psi_{1}\right) \psi$ is a nondecreasing function;

$\left(\psi_{2}\right) \sum_{n=1}^{\infty} \psi^{n}(t)<\infty$ for each $t>0$, where $\psi^{n}$ is the $n$th iterate of $\psi$.

Let us notice that the class $\Psi$ is nonempty. Indeed, the functions $\psi(t)=k t$ belong to $\Psi$ whenever $k \in[0,1)$. 
AJMS

27,2

Remark 2.13. [27] For each $\psi \in \Psi$, we see that the following assertions hold:

(1) $\lim _{n \rightarrow \infty} \psi^{n}(t)=0$, for all $t>0$;

(2) $\psi(t)<t$ for each $t>0$;

(3) $\psi(0)=0$.

Lemma 2.14. Let $(X, p)$ be a partial metric space. Let $\left(x_{n}\right)_{n=0}^{\infty} \subseteq X$ be a sequence and $\psi \in \Psi$ be such that

(1) $p\left(x_{0}, x_{1}\right)>0$

(2) $p\left(x_{n}, x_{n+1}\right) \leq \psi\left(p\left(x_{n-1}, x_{n}\right)\right)$, for each $n \in \mathbb{N}$.

Then $\left(x_{n}\right)_{n=0}^{\infty}$ is a 0-Cauchy sequence.

Proof. By hypothesis (2), we have

$$
p\left(x_{n}, x_{n+1}\right) \leq \psi\left(p\left(x_{n-1}, x_{n}\right)\right) \text {, for each } n \in \mathbb{N} .
$$

By repeated use of condition (2.1) and $\left(\psi_{1}\right)$, we get

$$
p\left(x_{n}, x_{n+1}\right) \leq \psi^{n}\left(p\left(x_{0}, x_{1}\right)\right) \text {, for each } n \in \mathbb{N} .
$$

For $m, n \in \mathbb{N}$ with $m>n$, we have

$$
\begin{aligned}
p\left(x_{n}, x_{m}\right) & \leq p\left(x_{n}, x_{n+1}\right)+p\left(x_{n+1}, x_{n+2}\right)+\ldots+p\left(x_{m-2}, x_{m-1}\right) \\
& +p\left(x_{m-1}, x_{m}\right) \leq \psi^{n}\left(p\left(x_{0}, x_{1}\right)\right)+\psi^{n+1}\left(p\left(x_{0}, x_{1}\right)\right)+\cdots+\psi^{m-2}\left(p\left(x_{0}, x_{1}\right)\right) \\
& +\psi^{m-1}\left(p\left(x_{0}, x_{1}\right)\right) \\
& =\sum_{i=n}^{m-1} \psi^{i}\left(p\left(x_{0}, x_{1}\right)\right) .
\end{aligned}
$$

Since $\sum_{n=1}^{\infty} \psi^{n}(t)<\infty$ for each $t>0$ and $p\left(x_{0}, x_{1}\right)>0$, it follows that

$$
\lim _{n, m \rightarrow \infty} p\left(x_{n}, x_{m}\right)=0 \text {. }
$$

This proves that $\left(x_{n}\right)_{n=0}^{\infty}$ is a 0 -Cauchy sequence in $X$.

\section{Main results}

In this section, we prove our new results. Throughout the paper, we use the following notation:

Let $(X, p)$ be a partial metric space, and $f, g: X \rightarrow X$ be self mappings. Then,

$$
M(g x, g y)=\max \left\{p(g x, g y), p(g x, f x), p(g y, f y), \frac{p(g x, f y)+p(g y, f x)}{2}\right\} .
$$

We begin with the following definition.

Definition 3.1. Let $X$ be a nonempty set, $q \in \mathbb{N}$, and let $f, g: X \rightarrow X$ be self- mappings. Then $X=\cup_{i=1}^{q} A_{i}$ is a cyclic representation of $X$ with respect to the pair $(f, g)$ if

(1) $A_{i}, i=1,2, \ldots, q$ are nonempty subsets of $X$;

(2) $f\left(A_{i}\right) \subseteq g\left(A_{i+1}\right)$ for $i=1,2, \ldots, q$, where $A_{q+1}=A_{1}$. 
Theorem 3.2. Let $(X, p)$ be a 0 -complete partial metric space, $q \in \mathbb{N}$, and let $A_{1}, A_{2}, \cdots, A_{q}$ be nonempty subsets of $X$, and $X=\cup_{i=1}^{q} A_{i}$. Suppose $f, g: X \rightarrow X$ are self- mappings, $g\left(A_{1}\right), g\left(A_{2}\right), \ldots, g\left(A_{q}\right)$ are closed subsets of $(X, p)$ and $X=\cup_{i=1}^{q} A_{i}$ is a cyclic representation of $X$ with respect to the pair $(f, g)$. If there exists $\psi \in \Psi$ such that

$$
p(f x, f y) \leq \psi(M(g x, g y))
$$

for all $x, y \in X$ with $(g x, g y) \in g\left(A_{i}\right) \times g\left(A_{i+1}\right), i=1,2, \ldots, q$, where $A_{q+1}=A_{1}$, then $f$ and $g$ have a unique point of coincidence $u$ in $\cap_{i=1}^{q} g\left(A_{i}\right)$ with $p(u, u)=0$. Moreover, if $f$ and $g$ are weakly compatible, then $f$ and $g$ have a unique common fixed point in $\cap_{i=1}^{q} g\left(A_{i}\right)$.

Proof. Let $x_{0}$ be an arbitrary element of $X$. Then there exists $i_{0} \in\{1,2, \ldots, q\}$ such that $x_{0} \in A_{i_{0}}$. Since $f\left(A_{i 0}\right) \subseteq g\left(A_{i 0+1}\right)$, there exists $x_{1} \in A_{i_{0}+1}$ such that $g x_{1}=f x_{0}$. Continuing this process, we can construct a sequence $\left(x_{n}\right)$ such that $g x_{n}=f x_{n-1}, n=1,2,3, \ldots$, where $x_{n} \in A_{i_{0}+n}$ and $A_{q+k}=A_{k}$. If $p\left(g x_{n}, g x_{n+1}\right)=0$ for some $n \in \mathbb{N}$, then $g x_{n}=g x_{n+1}=f x_{n}$ and hence $g x_{n+1}$ is a point of coincidence of $f$ and $g$.

Without loss of generality, we may assume that

$$
p\left(g x_{n}, g x_{n+1}\right)>0, \forall n \in \mathbb{N} .
$$

We note that for all $n \in \mathbb{N}$ there exists $i \in\{1,2, \ldots, q\}$ such that $\left(x_{n}, x_{n+1}\right) \in A_{i} \times A_{i+1}$ and so, $\left(g x_{n}, g x_{n+1}\right) \in g\left(A_{i}\right) \times g\left(A_{i+1}\right)$. We first compute $M\left(g x_{n-1}, g x_{n}\right)$. We have,

$$
\begin{aligned}
& M\left(g x_{n-1}, g x_{n}\right)=\max \left\{\begin{array}{c}
p\left(g x_{n-1}, g x_{n}\right), p\left(g x_{n-1}, g x_{n}\right), p\left(g x_{n}, g x_{n+1}\right), \\
\frac{p\left(g x_{n-1}, g x_{n+1}\right)+p\left(g x_{n}, g x_{n}\right)}{2}
\end{array}\right\} \\
& \leq \max \left\{\begin{array}{l}
p\left(g x_{n-1}, g x_{n}\right), p\left(g x_{n}, g x_{n+1}\right), \\
\frac{p\left(g x_{n-1}, g x_{n}\right)+p\left(g x_{n}, g x_{n+1}\right)}{2}
\end{array}\right\} \\
& =\max \left\{p\left(g x_{n-1}, g x_{n}\right), p\left(g x_{n}, g x_{n+1}\right)\right\} \text {. }
\end{aligned}
$$

By $\left(\psi_{1}\right)$, it follows that

$$
\psi\left(M\left(g x_{n-1}, g x_{n}\right)\right) \leq \psi\left(\max \left\{p\left(g x_{n-1}, g x_{n}\right), p\left(g x_{n}, g x_{n+1}\right)\right\}\right) .
$$

For any natural number $n$, we have by applying conditions (3.1) and (3.2) that

$$
\begin{aligned}
p\left(g x_{n}, g x_{n+1}\right) & =p\left(f x_{n-1}, f x_{n}\right) \\
& \leq \psi\left(M\left(g x_{n-1}, g x_{n}\right)\right) \\
& \leq \psi\left(\max \left\{p\left(g x_{n-1}, g x_{n}\right), p\left(g x_{n}, g x_{n+1}\right)\right\}\right) .
\end{aligned}
$$

We shall show that $\left(g x_{n}\right)$ is a Cauchy sequence in $g(X)$.

If $\max \left\{p\left(g x_{n-1}, g x_{n}\right), p\left(g x_{n}, g x_{n+1}\right)\right\}=p\left(g x_{n}, g x_{n+1}\right)$, then from condition (3.3) and using $\psi(t)<t$ for each $t>0$, we obtain

$$
p\left(g x_{n}, g x_{n+1}\right) \leq \psi\left(p\left(g x_{n}, g x_{n+1}\right)\right)<p\left(g x_{n}, g x_{n+1}\right),
$$

which is a contradiction. Therefore,

$$
\max \left\{p\left(g x_{n-1}, g x_{n}\right), p\left(g x_{n}, g x_{n+1}\right)\right\}=p\left(g x_{n-1}, g x_{n}\right) .
$$


AJMS

27,2

176

Thus, we obtain from condition (3.3) that

$$
p\left(g x_{n}, g x_{n+1}\right) \leq \psi\left(p\left(g x_{n-1}, g x_{n}\right)\right), \text { for all } n \in \mathbb{N} .
$$

By using Lemma 2.14, it follows that $\left(g x_{n}\right)$ is a 0-Cauchy sequence in $g(X)$. Since $g(X)=\cup_{i=1}^{q} g\left(A_{i}\right)$, it follows that $g(X)$ is a closed subset of the 0-complete partial metric space $(X, p)$, and hence $g(X)$ is 0 -complete. So, $\left(g x_{n}\right)$ converges to some point $u \in g(X)$ such that $p(u, u)=0$. Therefore,

$$
\lim _{n \rightarrow \infty} p\left(g x_{n}, u\right)=p(u, u)=0
$$

We shall prove that $u \in \cap_{i=1}^{q} g\left(A_{i}\right)$.

As $x_{0} \in A_{i_{0}}$, it follows that the sequence $\left(g x_{n q}\right)_{n \geq 0} \subseteq g\left(A_{i_{0}}\right)$. Since $g\left(A_{i_{0}}\right)$ is closed, condition (3.4) ensures that $u \in g\left(A_{i_{0}}\right)$. Again, we get $\left(g x_{n q+1}\right)_{n \geq 0} \subseteq g\left(A_{i_{0}+1}\right)$, where $A_{q+k}=A_{k}$. Proceeding as above, we obtain that $u \in g\left(A_{i_{0}+1}\right)$. Continuing in this way, we get

$$
u \in \cap_{i=1}^{q} g\left(A_{i}\right) .
$$

Now we shall show that $u$ is a point of coincidence of $f$ and $g$.

Indeed, since $u \in g(X)$, there exists $t \in X$ such that $g t=u$. Now, if $x_{n} \in A_{i}$, then $\left(g x_{n}, g t\right)=\left(g x_{n}, u\right) \in g\left(A_{i}\right) \times g\left(A_{i+1}\right)$ because $u \in \cap_{i=1}^{q} g\left(A_{i}\right)$. Therefore $M\left(g x_{n}, g t\right)$ is well defined, and by applying (3.1), we obtain that for all $n \in \mathbb{N}$,

$$
p\left(g x_{n+1}, f t\right)=p\left(f x_{n}, f t\right) \leq \psi\left(M\left(g x_{n}, g t\right)\right),
$$

where

$$
\begin{aligned}
M\left(g x_{n}, g t\right) & =\max \left\{\begin{array}{l}
p\left(g x_{n}, g t\right), p\left(g x_{n}, f x_{n}\right), p(g t, f t), \\
\frac{p\left(g x_{n}, f t\right)+p\left(g t, f x_{n}\right)}{2}
\end{array}\right\} \\
& =\max \left\{\begin{array}{l}
p\left(g x_{n}, g t\right), p\left(g x_{n}, g x_{n+1}\right), p(g t, f t), \\
\frac{p\left(g x_{n}, f t\right)+p\left(g t, g x_{n+1}\right)}{2}
\end{array}\right\} .
\end{aligned}
$$

Suppose that $p(g t, f t) \neq 0$. Let $\epsilon=\frac{p(g t, f t)}{2}>0$. Since $\lim _{n \rightarrow \infty} p\left(g x_{n}, g t\right)=0$, there exists $k \in \mathbb{N}$ such that

$$
p\left(g x_{n}, g t\right)<\epsilon, \text { for each } n \geq k
$$

Then, for each $n \geq k$

$$
\begin{aligned}
p\left(g x_{n}, f t\right) & \leq p\left(g x_{n}, g t\right)+p(g t, f t)-p(g t, g t) \\
& \leq p\left(g x_{n}, g t\right)+p(g t, f t) \\
& <3 \epsilon .
\end{aligned}
$$

For $n \geq k$, we have

$$
\frac{p\left(g x_{n}, f t\right)+p\left(g t, g x_{n+1}\right)}{2}<\frac{1}{2}(3 \epsilon+\epsilon)=2 \epsilon .
$$


Moreover, for $n \geq k$, we have

$$
p\left(g x_{n}, g x_{n+1}\right) \leq p\left(g x_{n}, g t\right)+p\left(g t, g x_{n+1}\right)<2 \epsilon .
$$

Thus, for $n \geq k$, it follows from conditions (3.7), (3.8) and (3.9) that

Generalized cylic contractions

$$
\max \left\{\begin{array}{l}
p\left(g x_{n}, g t\right), p\left(g x_{n}, g x_{n+1}\right), p(g t, f t), \\
\frac{p\left(g x_{n}, f t\right)+p\left(g t, g x_{n+1}\right)}{2}
\end{array}\right\}=2 \epsilon=p(g t, f t) .
$$

Therefore, we obtain from (3.6) that

$$
p\left(g x_{n+1}, f t\right) \leq \psi(p(g t, f t)), \text { for each } n \geq k .
$$

By using condition (3.10), for $n \geq k$, we have

$$
\begin{aligned}
p(g t, f t) & \leq p\left(g t, g x_{n+1}\right)+p\left(g x_{n+1}, f t\right)-p\left(g x_{n+1}, g x_{n+1}\right) \\
& \leq p\left(g t, g x_{n+1}\right)+\psi(p(g t, f t)) .
\end{aligned}
$$

Passing to the limit as $n \rightarrow \infty$, we get

$$
p(g t, f t) \leq \psi(p(g t, f t)),
$$

which is a contradiction since $\psi(t)<t$ for each $t>0$. Therefore, $p(g t, f t)=0$ and hence $f t=g t=u$. Therefore, $u$ is a point of coincidence of $f$ and $g$ such that $u \in \cap_{i=1}^{q} g\left(A_{i}\right)$ and $p(u, u)=0$.

For uniqueness, we assume that there is another point of coincidence $v$ of $f$ and $g$ such that $v \in \cap_{i=1}^{q} g\left(A_{i}\right)$ and $p(v, v)=0$. By supposition, there exists $x \in X$ satisfying $v=g x=f x$. Since $u, v \in \cap_{i=1}^{q} g\left(A_{i}\right)$ and $g x=v, g t=u, M(g x, g y)$ is well defined, therefore applying (3.1), we have

$$
\begin{aligned}
p(u, v) & =p(f t, f x) \\
& \leq \psi\left(\max \left\{p(g t, g x), p(g t, f t), p(g x, f x), \frac{p(g t, f x)+p(g x, f t)}{2}\right\}\right) \\
& =\psi\left(\max \left\{p(u, v), p(u, u), p(v, v), \frac{p(u, v)+p(v, u)}{2}\right\}\right) \\
& =\psi(p(u, v)) .
\end{aligned}
$$

If $p(u, v)>0$, then from condition (3.11), we get

$$
0<p(u, v) \leq \psi(p(u, v))
$$

which is a contradiction since $\psi(t)<t$ for each $t>0$. So, it must be the case that $p(u, v)=0$, and hence $u=v$. Thus, $f$ and $g$ have a unique point of coincidence $u \in \cap_{i=1}^{q} g\left(A_{i}\right)$ and $p(u, u)=0$.

If $f$ and $g$ are weakly compatible, then by proposition $2.12, f$ and $g$ have a unique common fixed point in $\cap_{i=1}^{q} g\left(A_{i}\right)$.

Corollary 3.3. Let $(X, p)$ be a 0 -complete partial metric space, and let $f, g: X \rightarrow X$ be such that $f(X) \subseteq g(X)$ and $g(X)$ a closed subset of $(X, p)$. If there exists $\psi \in \Psi$ such that

$$
p(f x, f y) \leq \psi(M(g x, g y))
$$

for all $x, y \in X$, then $f$ and $g$ have a unique point of coincidence $u$ in $g(X)$ such that $p(u, u)=0$. 
AJMS

27,2

178

Moreover, if $f$ and $g$ are weakly compatible, then $f$ and $g$ have a unique common fixed point in $g(X)$.

Proof. The proof follows from Theorem 3.2 by taking $A_{1}=A_{2}=\cdots=A_{q}=X$.

Corollary 3.4. Let $(X, p)$ be a 0 -complete partial metric space, and let $f: X \rightarrow X$ be a mapping. Suppose there exists $\psi \in \Psi$ such that

$$
p(f x, f y) \leq \psi\left(\max \left\{p(x, y), p(x, f x), p(y, f y), \frac{p(x, f y)+p(y, f x)}{2}\right\}\right)
$$

for all $x, y \in X$. Then $f$ has a unique fixed point $u$ in $X$ such that $p(u, u)=0$.

Proof. Since the maps $f$ and $I$, the identity map on $X$ are weakly compatible, the proof follows from Theorem 3.2 by taking $A_{1}=A_{2}=\cdots=A_{q}=X$ and $g=I$.

Corollary 3.5. Let $(X, p)$ be a 0 -complete partial metric space, $q \in \mathbb{N}$, and let $A_{1}, A_{2}, \cdots, A_{q}$ be nonempty subsets of $X$, and $X=\cup_{i=1}^{q} A_{i}$. Suppose $f, g: X \rightarrow X$ are self-mappings, $g\left(A_{1}\right), g\left(A_{2}\right), \cdots, g\left(A_{q}\right)$ are closed subsets of $(X, p)$ and $X=\cup_{i=1}^{q} A_{i}$ is a cyclic representation of $X$ with respect to the pair $(f, g)$. If there exists $k \in[0,1)$ such that

$$
p(f x, f y) \leq k \max \left\{p(g x, g y), p(g x, f x), p(g y, f y), \frac{p(g x, f y)+p(g y, f x)}{2}\right\}
$$

for all $x, y \in X$ with $(g x, g y) \in g\left(A_{i}\right) \times g\left(A_{i+1}\right), i=1,2, \cdots, q$, where $A_{q+1}=A_{1}$, then $f$ and $g$ have a unique point of coincidence $u$ in $\cap_{i=1}^{q} g\left(A_{i}\right)$ with $p(u, u)=0$. Moreover, if $f$ and $g$ are weakly compatible, then $f$ and $g$ have a unique common fixed point in $\cap_{i=1}^{q} g\left(A_{i}\right)$.

Proof. The proof follows from Theorem 3.2 by taking $\psi(t)=k t$ for each $t \geq 0$, where $k \in[0,1)$ is a fixed number.

Corollary 3.6. Let $(X, p)$ be a 0 -complete partial metric space and $f: X \rightarrow X$ be a mapping. If there exists $k \in[0,1)$ such that

$$
p(f x, f y) \leq k \max \left\{p(x, y), p(x, f x), p(y, f y), \frac{p(x, f y)+p(y, f x)}{2}\right\}
$$

for all $x, y \in X$, then $f$ has a unique fixed point $u$ in $X$ with $p(u, u)=0$.

Proof. The result follows from Theorem 3.2 by taking $A_{1}=A_{2}=\cdots=A_{q}=X, g=I$ and $\psi(t)=k t$ for each $t \geq 0$, where $k \in[0,1)$ is a fixed number.

Corollary 3.7. Let $(X, p)$ be a 0 -complete partial metric space, $q \in \mathbb{N}$, and let $A_{1}, A_{2}, \cdots, A_{q}$ be nonempty subsets of $X, X=\cup_{i=1}^{q} A_{i}$. Suppose $f, g: X \rightarrow X$ are selfmappings, $g\left(A_{1}\right), g\left(A_{2}\right), \cdots, g\left(A_{q}\right)$ are closed subsets of $(X, p)$ and $X=\cup_{i=1}^{q} A_{i}$ is a cyclic representation of $X$ with respect to the pair $(f, g)$. If there exists $\alpha, \beta, \gamma, \delta \geq 0$ with $\alpha+\beta+\gamma+2 \delta<1$ such that

$$
p(f x, f y) \leq \alpha p(g x, g y)+\beta p(g x, f x)+\gamma p(g y, f y)+\delta(p(g x, f y)+p(g y, f x))
$$

for any $(g x, g y) \in g\left(A_{i}\right) \times g\left(A_{i+1}\right), i=1,2, \cdots, q$ with $A_{q+1}=A_{1}$, then $f$ and $g$ have a unique point of coincidence $u$ in $\cap_{i=1}^{q} g\left(A_{i}\right)$ with $p(u, u)=0$. Moreover, if $f$ and $g$ are weakly compatible, then $f$ and $g$ have a unique common fixed point in $\cap_{i=1}^{q} g\left(A_{i}\right)$. 
Proof. From condition (3.12), we obtain

$$
\begin{aligned}
p(f x, f y) & \leq \alpha p(g x, g y)+\beta p(g x, f x)+\gamma p(g y, f y)+\delta(p(g x, f y)+p(g y, f x)) \\
& \leq(\alpha+\beta+\gamma+2 \delta) M(g x, g y) \\
& =k M(g x, g y),
\end{aligned}
$$

where $k=(\alpha+\beta+\gamma+2 \delta) \in[0,1)$. Now, corollary 3.5 can be applied to obtain the desired result.

Remark 3.8. It is worth mentioning that theorem 3.8 [28] can be obtained as a particular case of Theorem 3.2. Moreover, we obtain various important fixed-point results in partial metric spaces including Matthews version of Banach contraction theorem [1] as a special case of corollary 3.7.

We now present our second main theorem

Theorem 3.9. Let $(X, p)$ be a 0 -complete partial metric space, and let $f, T: X \rightarrow X$ be mappings. Suppose there exists $\psi \in \Psi$ such that

$$
p(f x, T y) \leq \psi(N(x, y))
$$

for all $x, y \in X$, where $N(x, y)=\max \left\{p(x, y), p(x, f x), p(y, T y), \frac{p(x, T y)+p(y, f x)}{2}\right\}$. Then $f$ and $T$ have a unique common fixed point $u$ in $X$ with $p(u, u)=0$.

Proof. We first prove that $u$ is a fixed point of $T$ if and only if $u$ is a fixed point of $f$.

Suppose that $u$ is a fixed point of $T$, that is, $T u=u$. Then, by using condition (3.13), we obtain

$$
\begin{aligned}
p(f u, u) & =p(f u, T u) \\
& \leq \psi(N(u, u)),
\end{aligned}
$$

where

$$
\begin{aligned}
N(u, u) & =\max \left\{p(u, u), p(u, f u), p(u, T u), \frac{p(u, T u)+p(u, f u)}{2}\right\} \\
& =\max \left\{p(u, u), p(u, f u), \frac{p(u, u)+p(u, f u)}{2}\right\} \\
& =\max \{p(u, u), p(u, f u)\} \\
& =p(u, f u) .
\end{aligned}
$$

Therefore,

$$
p(f u, u) \leq \psi(p(u, f u)) .
$$

If $p(u, f u)>0$, then $\psi(p(u, f u))<p(u, f u)$, a contradiction. This gives that $p(u, f u)=0$ and hence $f u=u$. of $T$.

Proceeding similarly, we can show that if $u$ is a fixed point of $f$, then $u$ is also a fixed point

Let $x_{0} \in X$ be arbitrary. We can construct a sequence $\left(x_{n}\right)$ in $X$ such that

$$
x_{n}=\left\{\begin{array}{l}
f x_{n-1}, \text { if } n \text { is odd } \\
T x_{n-1}, \text { if } n \text { is even }
\end{array}\right.
$$


AJMS

27,2

180

We assume that $x_{n} \neq x_{n-1}$ for every $n \in \mathbb{N}$. If $x_{2 n}=x_{2 n+1}$ for some $n \in \mathbb{N} \cup\{0\}$, then $x_{2 n}=f x_{2 n}$ and hence $x_{2 n}$ is a fixed point of $f$. By our previous discussion, it follows that $x_{2 n}$ is also a fixed point of $T$. So, $x_{2 n}$ becomes a common fixed point of $f$ and $T$. The case $x_{2 n+1}=x_{2 n+2}$ for some $n \in \mathbb{N} \cup\{0\}$ can be treated similarly to achieve our goal. Therefore, $p\left(x_{n}, x_{n-1}\right)>0, \forall n \in \mathbb{N}$.

By using condition (3.13), we obtain

$$
p\left(x_{2 n+1}, x_{2 n+2}\right)=p\left(f x_{2 n}, T x_{2 n+1}\right) \leq \psi\left(N\left(x_{2 n}, x_{2 n+1}\right)\right),
$$

where

$$
\begin{aligned}
N\left(x_{2 n}, x_{2 n+1}\right) & =\max \left\{\begin{array}{l}
p\left(x_{2 n}, x_{2 n+1}\right), p\left(x_{2 n}, f x_{2 n}\right), p\left(x_{2 n+1}, T x_{2 n+1}\right), \\
\frac{p\left(x_{2 n}, T x_{2 n+1}\right)+p\left(x_{2 n+1}, f x_{2 n}\right)}{2}
\end{array}\right\} \\
& =\max \left\{\begin{array}{l}
p\left(x_{2 n}, x_{2 n+1}\right), p\left(x_{2 n+1}, x_{2 n+2}\right), \\
\frac{p\left(x_{2 n}, x_{2 n+2}\right)+p\left(x_{2 n+1}, x_{2 n+1}\right)}{2}
\end{array}\right\} \\
& \leq \max \left\{\begin{array}{l}
p\left(x_{2 n}, x_{2 n+1}\right), p\left(x_{2 n+1}, x_{2 n+2}\right), \\
\frac{p\left(x_{2 n}, x_{2 n+1}\right)+p\left(x_{2 n+1}, x_{2 n+2}\right)}{2}
\end{array}\right\} \\
& =\max \left\{p\left(x_{2 n}, x_{2 n+1}\right), p\left(x_{2 n+1}, x_{2 n+2}\right)\right\} .
\end{aligned}
$$

By using $\left(\psi_{1}\right)$, it follows from (3.14) that

$$
p\left(x_{2 n+1}, x_{2 n+2}\right) \leq \psi\left(\max \left\{p\left(x_{2 n}, x_{2 n+1}\right), p\left(x_{2 n+1}, x_{2 n+2}\right)\right\}\right) .
$$

If $\max \left\{p\left(x_{2 n}, x_{2 n+1}\right), p\left(x_{2 n+1}, x_{2 n+2}\right)\right\}=p\left(x_{2 n+1}, x_{2 n+2}\right)$, then by using $\psi(t)<t$ for each $t>0$, we obtain from condition (3.15) that

$$
p\left(x_{2 n+1}, x_{2 n+2}\right) \leq \psi\left(p\left(x_{2 n+1}, x_{2 n+2}\right)\right)<p\left(x_{2 n+1}, x_{2 n+2}\right),
$$

which is a contradiction. Therefore,

$$
\max \left\{p\left(x_{2 n}, x_{2 n+1}\right), p\left(x_{2 n+1}, x_{2 n+2}\right)\right\}=p\left(x_{2 n}, x_{2 n+1}\right) .
$$

Thus, condition (3.15) becomes

$$
p\left(x_{2 n+1}, x_{2 n+2}\right) \leq \psi\left(p\left(x_{2 n}, x_{2 n+1}\right)\right), \forall n \in \mathbb{N} .
$$

Similarly, we can show that

$$
p\left(x_{2 n}, x_{2 n+1}\right) \leq \psi\left(p\left(x_{2 n-1}, x_{2 n}\right)\right), \forall n \in \mathbb{N} .
$$

Combining conditions (3.16) and (3.17), we get

$$
p\left(x_{n}, x_{n+1}\right) \leq \psi\left(p\left(x_{n-1}, x_{n}\right)\right), \forall n \in \mathbb{N} .
$$

By using Lemma 2.14, it follows that $\left(x_{n}\right)$ is a 0-Cauchy sequence in $X$. As $(X, p)$ is 0-complete, there exists $u \in X$ such that $x_{n} \rightarrow u$ with $p(u, u)=0$, that is, $\lim _{n \rightarrow \infty} p\left(x_{n}, u\right)=p(u, u)=0$. This ensures that $\lim _{n \rightarrow \infty} p\left(x_{2 n}, u\right)=p(u, u)=0$ and $\lim _{n \rightarrow \infty} p\left(x_{2 n+1}, u\right)=p(u, u)=0$. 
By using condition (3.13), we obtain

$$
p\left(x_{2 n+1}, T u\right)=p\left(f x_{2 n}, T u\right) \leq \psi\left(N\left(x_{2 n}, u\right)\right),
$$

where

$$
\begin{aligned}
N\left(x_{2 n}, u\right) & =\max \left\{\begin{array}{l}
p\left(x_{2 n}, u\right), p\left(x_{2 n}, f x_{2 n}\right), p(u, T u), \\
\frac{p\left(x_{2 n}, T u\right)+p\left(u, f x_{2 n}\right)}{2}
\end{array}\right\} \\
& =\max \left\{\begin{array}{l}
p\left(x_{2 n}, u\right), p\left(x_{2 n}, x_{2 n+1}\right), p(u, T u), \\
\frac{p\left(x_{2 n}, T u\right)+p\left(u, x_{2 n+1}\right)}{2}
\end{array}\right\} .
\end{aligned}
$$

Generalized

cylic

contractions Suppose that $p(u, T u) \neq 0$. Let $\epsilon=\frac{p(u, T u)}{2}>0$. Since $\lim _{n \rightarrow \infty} p\left(x_{2 n}, u\right)=0$, there exists $k_{1} \in \mathbb{N}$
such that

$$
p\left(x_{2 n}, u\right)<\epsilon \text {, for each } n \geq k_{1} \text {. }
$$

Then, for each $n \geq k_{1}$

$$
\begin{aligned}
p\left(x_{2 n}, T u\right) & \leq p\left(x_{2 n}, u\right)+p(u, T u)-p(u, u) \\
& =p\left(x_{2 n}, u\right)+p(u, T u) \\
& <3 \epsilon .
\end{aligned}
$$

As $\lim _{n \rightarrow \infty} p\left(x_{2 n+1}, u\right)=0$, there exists $k_{2} \in \mathbb{N}$ such that

$$
p\left(x_{2 n+1}, u\right)<\epsilon, \text { for each } n \geq k_{2} \text {. }
$$

Put $k=\max \left\{k_{1}, k_{2}\right\}$. Then, for $n \geq k$, we have

$$
\frac{p\left(x_{2 n}, T u\right)+p\left(u, x_{2 n+1}\right)}{2}<\frac{1}{2}(3 \epsilon+\epsilon)=2 \epsilon .
$$

Moreover, for $n \geq k$, we have

$$
p\left(x_{2 n}, x_{2 n+1}\right) \leq p\left(x_{2 n}, u\right)+p\left(u, x_{2 n+1}\right)<2 \epsilon .
$$

Thus, for $n \geq k$, it follows from conditions (3.20), (3.21) and (3.22) that

$$
\max \left\{\begin{array}{l}
p\left(x_{2 n}, u\right), p\left(x_{2 n}, x_{2 n+1}\right), p(u, T u), \\
\frac{p\left(x_{2 n}, T u\right)+p\left(u, x_{2 n+1}\right)}{2}
\end{array}\right\}=2 \epsilon=p(u, T u) .
$$

Therefore, we obtain from (3.19) that

$$
p\left(x_{2 n+1}, T u\right) \leq \psi(p(u, T u)), \text { for each } n \geq k .
$$

By using condition (3.23), for $n \geq k$, we have

$$
\begin{aligned}
p(u, T u) & \leq p\left(u, x_{2 n+1}\right)+p\left(x_{2 n+1}, T u\right)-p\left(x_{2 n+1}, x_{2 n+1}\right) \\
& \leq p\left(u, x_{2 n+1}\right)+\psi(p(u, T u)) .
\end{aligned}
$$

Passing to the limit as $n \rightarrow \infty$ and using Lemma $2.8(a)$, we get

$$
p(u, T u) \leq \psi(p(u, T u)),
$$


AJMS

27,2

182

which is a contradiction, since $\psi(t)<t$ for each $t>0$. Therefore, $p(u, T u)=0$ and hence $T u=u$. This proves that $u$ is a fixed point of $T$. By our previous discussion, $u$ is also a fixed point of $f$. Thus, $u$ is a common fixed point of $f$ and $T$ in $X$ with $p(u, u)=0$.

For uniqueness, let $v$ be another common fixed point of $f$ and $T$ in $X$ with $p(v, v)=0$. By applying condition (3.13), we get

$$
p(u, v)=p(f u, T v) \leq \psi(N(u, v)),
$$

where

$$
\begin{aligned}
N(u, v) & \left.=\max \{p(u, v), p(u, f u), p(v, T v)\}, \frac{p(u, T v)+p(v, f u)}{2}\right\} \\
& =\max \{p(u, v), 0,0, p(u, v)\} \\
& =p(u, v) .
\end{aligned}
$$

Thus, condition (3.24) becomes

$$
p(u, v) \leq \psi(p(u, v))
$$

If $p(u, v)>0$, then

$$
0<p(u, v) \leq \psi(p(u, v))
$$

which is a contradiction since $\psi(t)<t$ for each $t>0$. So, it must be the case that $p(u, v)=0$ and hence $u=v$. Therefore, $u$ is a unique common fixed point of $f$ and $T$ in $X$ with $p(u, u)=0$.

Corollary 3.10. Let $(X, p)$ be a 0 -complete partial metric space, and let the mappings $f, T: X \rightarrow X$ be such that

$$
p(f x, T y) \leq k \max \left\{p(x, y), p(x, f x), p(y, T y), \frac{p(x, T y)+p(y, f x)}{2}\right\}
$$

for all $x, y \in X$. where $k \in[0,1)$ is a constant. Then $f$ and $T$ have a unique common fixed point $u$ in $X$ with $p(u, u)=0$.

Proof. The proof follows from Theorem 3.9 by taking $\psi(t)=k t$ for each $t \geq 0$, where $k \in[0,1)$ is a fixed number.

Corollary 3.11. Let $(X, p)$ be a 0 -complete partial metric space, and let $f: X \rightarrow X$ be a mapping. Suppose there exists $\psi \in \Psi$ such that

$$
p(f x, f y) \leq \psi\left(N^{\prime}(x, y)\right)
$$

for all $x, y \in X$, where $N^{\prime}(x, y)=\max \left\{p(x, y), p(x, f x), p(y, f y), \frac{p(x, f y)+p(y, f x)}{2}\right\}$. Then $f$ has a unique fixed point $u$ in $X$ with $p(u, u)=0$.

Proof. The proof follows from Theorem 3.9 by considering $T=f$.

Corollary 3.12. Let $(X, p)$ be a 0 -complete partial metric space, and let the mappings $f, T: X \rightarrow X$ be such that

$$
p(f x, T y) \leq \alpha p(x, y)+\beta p(x, f x)+\gamma p(y, T y)+\delta(p(x, T y)+p(y, f x))
$$

for all $x, y \in X$, where $\alpha, \beta, \gamma, \delta \geq 0$ with $\alpha+\beta+\gamma+2 \delta<1$. Then $f$ and $T$ have a unique common fixed point $u$ in $X$ with $p(u, u)=0$. 
Proof. From condition (3.25), we obtain

$$
\begin{aligned}
p(f x, T y) & \leq \alpha p(x, y)+\beta p(x, f x)+\gamma p(y, T y)+\delta(p(x, T y)+p(y, f x)) \\
& \leq(\alpha+\beta+\gamma+2 \delta) N(x, y) \\
& =k N(x, y) \\
& =\psi(N(x, y)),
\end{aligned}
$$

where $k=(\alpha+\beta+\gamma+2 \delta) \in[0,1)$ and $\psi(t)=k t$ for each $t \geq 0$. Now applying Theorem 3.9, we can obtain the desired result.

Remark 3.13. The results of this study are obtained under the weaker assumption that the underlying partial metric space is 0-complete. However, they also valid if the space is complete.

Finally we conclude this section by providing two applications of our main results.

Example 3.14. Let $X=\left\{\left[3-5^{-n}, 3\right]: n \in \mathbb{N}\right\} \cup\left\{\left[3,3+5^{-n}\right]: n \in \mathbb{N}\right\} \cup\{\{3\}\}$, where $\{3\}=[3,3]$. We define $p: X \times X \rightarrow \mathbb{R}^{+}$by $p([a, b],[c, d])=\max \{b, d\}-\min \{a, c\}$. Then $(X, p)$ is a 0 -complete partial metric space. Let $A_{1}=\left\{\left[3-5^{-n}, 3\right]: n \in \mathbb{N}\right\} \cup\{\{3\}\}$ and $A_{2}=\left\{\left[3,3+5^{-n}\right]: n \in \mathbb{N}\right\} \cup\{\{3\}\}$. Obviously, $X=A_{1} \cup A_{2}$. Define mappings $f, g: X \rightarrow X$ by

$$
f x=\left\{\begin{array}{l}
{\left[3,3+5^{-(n+2)}\right], \text { if } x=\left[3-5^{-n}, 3\right]} \\
{\left[3-5^{-(n+2)}, 3\right], \text { if } x=\left[3,3+5^{-n}\right]} \\
\{3\}, \text { if } x=\{3\}
\end{array}\right.
$$

and

$$
g x=\left\{\begin{array}{l}
{\left[3-5^{-(n+1)}, 3\right], \text { if } x=\left[3-5^{-n}, 3\right]} \\
{\left[3,3+5^{-(n+1)}\right], \text { if } x=\left[3,3+5^{-n}\right]} \\
\{3\}, \text { if } x=\{3\}
\end{array}\right.
$$

Then, $f\left(A_{1}\right) \subseteq g\left(A_{2}\right), f\left(A_{2}\right) \subseteq g\left(A_{1}\right)$ and so $X=A_{1} \cup A_{2}$ is a cyclic representation of $X$ with respect to the pair $(f, g)$. Moreover, $g\left(A_{1}\right), g\left(A_{2}\right)$ are closed subsets of $(X, p)$. We now verify condition (3.1) with the control function $\psi:[0, \infty) \rightarrow[0, \infty)$ given by $\psi(t)=\frac{t}{3}$. We now consider the following cases:

Case-I. $x=\left[3-5^{-n}, 3\right] \in A_{1}, y=\left[3,3+5^{-k}\right] \in A_{2}, n, k \in \mathbb{N}$ with $n<k$. In this case, we have $5^{-k}<5^{-n}$ and $5^{-k} \leq 5^{-(n+1)}$. Then,

$$
\begin{gathered}
p(f x, f y)=p\left(\left[3,3+5^{-(n+2)}\right],\left[3-5^{-(k+2)}, 3\right]\right)=\frac{1}{25}\left(5^{-n}+5^{-k}\right)<\frac{2}{25} \cdot 5^{-n}, \\
p(g x, g y)=p\left(\left[3-5^{-(n+1)}, 3\right],\left[3,3+5^{-(k+1)}\right]\right)=5^{-(k+1)}+5^{-(n+1)} \\
=\frac{1}{5} \cdot 5^{-k}+5^{-(n+1)} \leq\left(\frac{1}{5}+1\right) 5^{-(n+1)}=\frac{6}{25} \cdot 5^{-n}, \\
p(g x, f x)=p\left(\left[3-5^{-(n+1)}, 3\right],\left[3,3+5^{-(n+2)}\right]\right)=5^{-(n+2)}+5^{-(n+1)}=\frac{6}{25} \cdot 5^{-n}, \\
p(g y, f y)=p\left(\left[3,3+5^{-(k+1)}\right],\left[3-5^{-(k+2)}, 3\right]\right)=5^{-(k+1)}+5^{-(k+2)}<\frac{6}{25} \cdot 5^{-n},
\end{gathered}
$$


AJMS

27,2

$$
\begin{aligned}
& p(g x, f y)=p\left(\left[3-5^{-(n+1)}, 3\right],\left[3-5^{-(k+2)}, 3\right]\right)=5^{-(n+1)}=\frac{1}{5} \cdot 5^{-n}, \\
& p(f x, g y)=p\left(\left[3,3+5^{-(n+2)}\right],\left[3,3+5^{-(k+1)}\right]\right)=5^{-(n+2)}=\frac{1}{25} \cdot 5^{-n} .
\end{aligned}
$$

$184 \quad$ Now, $\quad \frac{p(g x, f y)+p(f x, g y)}{2}=\frac{1}{2}\left(\frac{1}{5} \cdot 5^{-n}+\frac{1}{25} \cdot 5^{-n}\right)=\frac{3}{25} \cdot 5^{-n}<\frac{6}{25} \cdot 5^{-n}$.

Thus, $M(g x, g y)=\frac{6}{25} \cdot 5^{-n}$. Therefore,

$$
p(f x, f y)<\frac{2}{25} \cdot 5^{-n}=\psi(M(g x, g y)) .
$$

Case-II. $x=\left[3-5^{-n}, 3\right] \in A_{1}, y=\left[3,3+5^{-k}\right] \in A_{2}, n, k \in \mathbb{N}$ with $n>k$.

In this case, we have $5^{-k}>5^{-n}$ and $5^{-n} \leq 5^{-(k+1)}$. Then, $p(f x, f y)<\frac{2}{25} \cdot 5^{-k}, p(g x, g y)=\frac{1}{5}$ $\left(5^{-k}+5^{-n}\right) \leq \frac{6}{25} \cdot 5^{-k}, p(g x, f x)=\frac{6}{25} \cdot 5^{-n}, p(g y, f y)=\frac{6}{25} \cdot 5^{-k}$ and $p(g x, f y)=5^{-(k+2)}=\frac{1}{25} \cdot 5^{-k}$, $p(f x, g y)=5^{-(k+1)}=\frac{1}{5} \cdot 5^{-k}$. So, $\frac{p(g x, f y)+p(f x, g y)}{2}=\frac{6}{25} \cdot 5^{-k}$.

Thus, $M(g x, g y)=\frac{6}{25} \cdot 5^{-k}$. Therefore,

$$
p(f x, f y)<\frac{2}{25} \cdot 5^{-k}=\psi(M(g x, g y)) .
$$

Case-III. $x=\left[3-5^{-n}, 3\right] \in A_{1}, y=\left[3,3+5^{-k}\right] \in A_{2}, n, k \in \mathbb{N}$ with $n=k$.

Then, $p(f x, f y)=\frac{2}{25} \cdot 5^{-n}, p(g x, g y)=\frac{2}{5} \cdot 5^{-n}, p(g x, f x)=\frac{6}{25} \cdot 5^{-n}, p(g y, f y)=\frac{6}{25} \cdot 5^{-n}$ and $p(g x, f y)=$ $\frac{1}{5} \cdot 5^{-n}, p(f x, g y)=\frac{1}{5} \cdot 5^{-n}$. So, $\frac{p(g x, f y)+p(f x, g y)}{2}=\frac{1}{5} \cdot 5^{-n}$.

Thus, $M(g x, g y)=\frac{6}{25} \cdot 5^{-n}$. Therefore,

$$
p(f x, f y)=\frac{2}{25} \cdot 5^{-n}=\psi(M(g x, g y)) .
$$

Case-IV. $x=\left[3-5^{-n}, 3\right] \in A_{1}, n \in \mathbb{N}, y=\{3\} \in A_{2}$.

Then,

$$
\begin{gathered}
p(f x, f y)=p\left(\left[3,3+5^{-(n+2)}\right],\{3\}\right)=5^{-(n+2)}=\frac{1}{25} \cdot 5^{-n}, \\
p(g x, g y)=p\left(\left[3-5^{-(n+1)}, 3\right],\{3\}\right)=5^{-(n+1)}=\frac{1}{5} \cdot 5^{-n}, \\
p(g x, f x)=p\left(\left[3-5^{-(n+1)}, 3\right],\left[3,3+5^{-(n+2)}\right]\right)=5^{-(n+2)}+5^{-(n+1)}=\frac{6}{25} \cdot 5^{-n}, \\
p(g y, f y)=p(\{3\},\{3\})=0, p(g x, f y)=p\left(\left[3-5^{-(n+1)}, 3\right],\{3\}\right)=\frac{1}{5} \cdot 5^{-n}, \\
p(f x, g y)=p\left(\left[3,3+5^{-(n+2)}\right],\{3\}\right)=\frac{1}{25} \cdot 5^{-n} . \text { Thus, } M(g x, g y)=\frac{6}{25} \cdot 5^{-n} . \\
\text { Therefore, } p(f x, f y)=\frac{1}{25} \cdot 5^{-n}<\frac{2}{25} \cdot 5^{-n}=\psi(M(g x, g y)) .
\end{gathered}
$$


Case-V. $x=\{3\} \in A_{1}, y=\left[3,3+5^{-n}\right] \in A_{2}, n \in \mathbb{N}$.

In this case, we have

$$
p(f x, f y)=\frac{1}{25} \cdot 5^{-n}, M(g x, g y)=\frac{6}{25} \cdot 5^{-n} .
$$

Therefore,

$$
p(f x, f y)=\frac{1}{25} \cdot 5^{-n}<\frac{2}{25} \cdot 5^{-n}=\psi(M(g x, g y)) .
$$

Case-VI. $x=y=\{3\}$ is trivial.

The other possibility is treated similarly. Moreover, $f$ and $g$ are weakly compatible. Thus, all the conditions of Theorem 3.2 are fulfilled, and $\{3\}$ is the unique common fixed point of $f$ and $g$ in $g\left(A_{1}\right) \cap g\left(A_{2}\right)$ with $p(\{3\},\{3\})=0$.

Even if in the following example it is not hard to prove that $\mathbf{0}$ is the only common fixed point, it can provide an alternative proof and can inspire other application of Theorem 3.9.

Example 3.15. Let $\beta \in(0,1)$ be fixed, and let $X=A \cup\{\mathbf{0}\} \subseteq \ell^{1}$, where $\mathbf{0}=(0)_{n=1}^{\infty}$ and the subset $A$ of $\ell^{1}$ defined by $x^{q}=\left(x_{n}^{q}\right)_{n=1}^{\infty} \in A$ if $f$

$$
x_{n}^{q}=\left\{\begin{array}{l}
0, \text { if } n<2 q, \text { or } n=2 k-1, k \in \mathbb{N}, \\
\beta^{n}, \text { if } n=2 k \geq 2 q
\end{array}\right.
$$

for $q=1,2,3, \cdots$. Define $p: X \times X \rightarrow \mathbb{R}^{+}$by $p\left(\left(x_{n}\right),\left(y_{n}\right)\right)=\sum_{n=1}^{\infty} \max \left\{x_{n}, y_{n}\right\}$ for all $\left(x_{n}\right),\left(y_{n}\right) \in X$. Then $(X, p)$ is a 0 -complete partial metric space. Let $T, f: X \rightarrow X$ be defined by

$$
T(\underbrace{0, \cdots, 0}_{2 q-1}, \beta^{2 q}, 0, \beta^{2 q+2}, 0, \cdots)=(\underbrace{0, \cdots, 0}_{2 q+1}, \beta^{2 q+2}, 0, \beta^{2 q+4}, 0, \cdots), T \mathbf{0}=\mathbf{0}
$$

and

$$
f(\underbrace{0, \cdots, 0}_{2 q-1}, \beta^{2 q}, 0, \beta^{2 q+2}, 0, \cdots)=(\underbrace{0, \cdots, 0}_{2 q+3}, \beta^{2 q+4}, 0, \beta^{2 q+6}, 0, \cdots), f \mathbf{0}=\mathbf{0} .
$$

Define $\psi:[0, \infty) \rightarrow[0, \infty)$ by $\psi(t)=\beta t$

We now verify condition (3.13) for all $x, y \in X$.

Case-I. Take $x=(\underbrace{0, \cdots, 0}_{2 q-1}, \beta^{2 q}, 0, \beta^{2 q+2}, 0, \cdots) \in A$ and

$$
y=(\underbrace{0, \cdots, 0}_{2 r-1}, \beta^{2 r}, 0, \beta^{2 r+2}, 0, \cdots) \in A .
$$


AJMS

27,2

186
Then

$$
\begin{aligned}
& f x=(\underbrace{0, \cdots, 0}_{2 q+3}, \beta^{2 q+4}, 0, \beta^{2 q+6}, 0, \cdots), \\
& T y=(\underbrace{0, \cdots, 0}_{2 r+1}, \beta^{2 r+2}, 0, \beta^{2 r+4}, 0, \cdots) .
\end{aligned}
$$

(1) If $r \leq q$, then

$$
\begin{gathered}
p(f x, T y)=\beta^{2 r+2}+\beta^{2 r+4}+\cdots+\beta^{2 q+2}+\beta^{2 q+4}+\beta^{2 q+6}+\cdots \\
\left.=\beta^{2}\left[\beta^{2 r}+\beta^{2 r+2}+\cdots+\beta^{2 q-2}+\beta^{2 q}\right]\right]+\frac{\beta^{2 q+4}}{1-\beta^{2}} \\
p(x, y)=\beta^{2 r}+\beta^{2 r+2}+\cdots+\beta^{2 q-2}+\beta^{2 q}+\beta^{2 q+2}+\beta^{2 q+4}+\cdots \\
=\beta^{2 r}+\beta^{2 r+2}+\cdots+\beta^{2 q-2}+\beta^{2 q}+\frac{\beta^{2 q+2}}{1-\beta^{2}} .
\end{gathered}
$$

Thus,

$$
p(f x, T y)=\beta^{2} p(x, y)<\beta p(x, y) \leq \beta N(x, y)=\psi(N(x, y)) .
$$

(2) If $r>q$, then $2 r+2>2 q+2 \Rightarrow 2 r+2 \geq 2 q+4$ and

$$
\begin{gathered}
p(f x, T y)=\beta^{2 q+4}+\beta^{2 q+6}+\cdots+\beta^{2 r}+\beta^{2 r+2}+\beta^{2 r+4}+\cdots \\
=\beta^{2 q+4}+\beta^{2 q+6}+\cdots+\beta^{2 r-2}+\beta^{2 r}+\frac{\beta^{2 r+2}}{1-\beta^{2}} \\
p(x, y)=\beta^{2 q}+\beta^{2 q+2}+\cdots+\beta^{2 r-2}+\beta^{2 r}+\beta^{2 r+2}+\beta^{2 r+4}+\cdots \\
=\beta^{2 q}+\beta^{2 q+2}+\cdots+\beta^{2 r-4}+\beta^{2 r-2}+\frac{\beta^{2 r}}{1-\beta^{2}}
\end{gathered}
$$

which implies that

$$
p(f x, T y)<\beta^{2} p(x, y)<\beta p(x, y) \leq \beta N(x, y)=\psi(N(x, y)) .
$$

Case-II. Take $x=(\underbrace{0, \cdots, 0}_{2 q-1}, \beta^{2 q}, 0, \beta^{2 q+2}, 0, \cdots) \in A$ and $y=\mathbf{0}$.
Then

$$
\begin{gathered}
f x=(\underbrace{0, \cdots, 0}_{2 q+3}, \beta^{2 q+4}, 0, \beta^{2 q+6}, 0, \cdots), T y=\mathbf{0}, \\
p(f x, T y)=\beta^{2 q+4}+\beta^{2 q+6}+\cdots=\frac{\beta^{2 q+4}}{1-\beta^{2}},
\end{gathered}
$$




$$
p(x, y)=\beta^{2 q}+\beta^{2 q+2}+\cdots=\frac{\beta^{2 q}}{1-\beta^{2}} .
$$

Thus,

$$
p(f x, T y)=\beta^{4} p(x, y)<\beta p(x, y) \leq \beta N(x, y)=\psi(N(x, y)) .
$$

The case $x=\mathbf{0}, y \in A$ can be treated similarly, and the case $x=y=\mathbf{0}$ is trivial.

Thus, we have all the conditions of Theorem 3.9, and $\mathbf{0}$ is the unique common fixed point of $f$ and $T$ in $X$ with $p(\mathbf{0}, \mathbf{0})=0$.

\section{References}

1. Matthews S. Partial metric topology. Ann N.Y. Acad Sci. 1994 (728): 183-97.

2. Altun I, Acar O. Fixed point theorems for weak contractions in the sense of Berinde on partial metric spaces. Topol Appl. 2012; 159: 2642-48.

3. Altun I, Sola F, Simsek H. Generalized contractions on partial metric spaces. Topol Appl. 2010; 157: 2778-85.

4. Bukatin M, Kopperman R, Matthews S, Pajoohesh H. Partial metric spaces. Am Math Mon. 2009; 116: 708-18.

5. Ciric L, Samet B, Aydi H, Vetro C. Common fixed points of generalized contractions on partial metric spaces and an application. Appl Math Comput. 2011; 218: 2398-2406.

6. Heckmann R. Approximation of metric spaces by partial metric spaces. Appl Categ. Struct. 1999; 7: 71-83.

7. Karapinar E. A note on common fixed point theorems in partial metric spaces. Miskolc Math Notes. 2011; 12: 185-91.

8. Mohanta SK. Common fixed point theorems via w-distance. Bull Math Anal Appl. 2011; 3: 182-89.

9. Mohanta SK, Mohanta S. A common fixed point theorem in G-metric spaces. Cubo, A Math J. 2012; 14: 85-101.

10. Mohanta SK, Patra S. Coincidence points and common fixed points for hybrid pair of mappings in b-metric spaces endowed with a graph. J Lin Top Alg. 2017; 6: 301-21.

11. Kirk WA, Srinivasan PS, Veeramani P. Fixed points for mappings satisfying cyclical contractive conditions. Fixed Point Theory. 2003; 4: 79-89.

12. Agarwal RP, Alghamdi MA, Shahzad N. Fixed point for cyclic generalized contractions in partial metric spaces. Fixed Point Theory Appl. 2012; 2012(40): 1-11.

13. Abbas M, Nazir T, Romaguera S. Fixed point results for generalized cyclic contraction mappings in partial metric spaces. Rev Real Acad Ciencias Exactas, Fis. Nat. 2012; 106: 287-97.

14. Bari CD, Vetro P. Fixed points for weak $\varphi$-contractions on partial metric spaces. Int J Contemp Math Sci. 2011; 1: 5-12.

15. Karapinar E, Yuce IS. Fixed point theory for cyclic generalized weak $\varphi$-contraction on partial metric spaces. Abs Appl Anal. 2012; 2012, 491542.

16. Karapinar E, Shobkolaei N, Sedghi S, Vaezpour SM. A common fixed point theorem for cyclic operators in partial metric spaces. Filomat. 2012; 26: 407-14.

17. Shatanawi W, Postolache M. Common fixed point results for mappings under nonlinear contraction of cyclic form in ordered metric spaces. Fixed Point Theory Appl. 2010; 2010, 493298.

18. Khan MS, Swaleh M, Sessa S.. Fixed point theorems by altering distances between the points. Bull Aust Math Soc. 1984; 30: 1-9. 
AJMS

27,2

188

19. He F, Chen A. Fixed points for cyclic $\phi$-contractions in generalized metric spaces. Fixed Point Theory Appl. 2016; 2016(67): 1-12.

20. Nashine HK, Kadelburg Z. Cyclic contractions and fixed point results via control functions on partial metric spaces. Int J Anal. 2013; 2013, 726387.

21. Pacurar M, Rus IA. Fixed point theory for cyclic $\phi$-contractions. Nonlinear Anal. 2010; 72: 1181-87.

22. Yamaod O, Sintunavarat W, Cho YJ. Common fixed point theorems for generalized cyclic contraction pairs in b-metric spaces with applications. Fixed Point Theory Appl. 2015; 2015: 164.

23. Romaguera S. A Kirk type characterization of completeness for partial metric spaces. Fixed Point Theory Appl. 2010; 2010: 493298.

24. Abdeljawad T, Karapinar E, Taş K. Existence and uniqueness of a common fixed point on partial metric spaces. Appl Math Lett. 2011; 24: 1900-04.

25. Jungck G. Common fixed points for noncontinuous nonself maps on non-metric spaces. Far East J Math Sci. 1996; 4: 199-215.

26. Abbas M, Jungck G. Common fixed point results for noncommuting mappings without continuity in cone metric spaces. J Math Anal Appl. 2008; 341: 416-20.

27. Kaushik P, Kumar S. Fixed point results for $(\alpha, \psi, \xi)$ contractive compatible multi-valued mappings. J Nonlinear Anal Appl. 2016; 2016(2): 28-36.

28. Samet B, Turinici M. Fixed point theorems on a metric space endowed with an arbitrary binary relation and applications. Commun Math Anal. 2012; 13: 82-97.

\section{Corresponding author}

Sushanta Kumar Mohanta can be contacted at: mohantawbsu@rediffmail.com

For instructions on how to order reprints of this article, please visit our website:

www.emeraldgrouppublishing.com/licensing/reprints.htm

Or contact us for further details: permissions@emeraldinsight.com 